\title{
Advances in Thermographic Signal Reconstruction
}

by S. Shepard

\author{
Thermal Wave Imaging, Inc., Ferndale, MI USA 48220, sshepard@thermalwave.com
}

\begin{abstract}
Since its introduction in 1999, the Thermographic Signal Reconstruction (TSR) method has become widely used for enhancement and analysis of sequence data. While TSR image data is often sufficient for many applications, examination of the resulting logarithmic time derivatives of each pixel have facilitated significant insight into the details of the active thermography process. The deterministic nature of the derivatives has facilitated the successful implementation of automated defect recognition, and perhaps more significantly, robust material characterization in a flaw-free sample. We present a summary of these advances, and the relationships between the TSR derivatives and the thermophysical properties of a sample.
\end{abstract}

\section{Introduction}

When first conceived in 1999, the Thermographic Signal Reconstruction (TSR) method was intended to enhance the subjective quality of images acquired from active thermography sequences by reducing the amount of temporal noise in each pixel time history. It went about this by fitting the logarithmic time evolution of each pixel with a low order polynomial function, and then transforming that function back to the linear domain. The result was a temporally low-pass filtered replica of the original data from the IR camera that was almost entirely free of temporal noise. Perhaps the most striking thing about this early test of TSR was that although it dramatically improved the temporal signal-to-noise characteristics of a given pixel, it had almost no effect on the feature-to-background content of the image. The lesson learned was that removal of temporal noise from the camera data sequence did not necessarily provide any benefit for the practice of Nondestructive Evaluation (NDE). Immediately following the initial (unsuccessful) attempt to improve IR NDE results using TSR, the researcher team recognized that the true benefit of the method was not in attempting to replicate the original data, but rather, to leave it in the logarithmic domain and view the derivatives of the logarithmic temperature-time evolution as images. The result is the modern implementation of TSR, which has become a widely accepted NDE tool, and has been discussed and analyzed extensively in the literature. It has become common practice to rely on the first and second TSR derivative images, and often, the original raw (or noise reduced) IR image is bypassed entirely. The derivative images offer significantly greater feature to background contrast, and a constrained dynamic range in which the derivative amplitudes are prescribed. Most importantly, they allow detection of deep or subtle features that may be undetectable in the original IR data sequence.

\section{TSR Derivative Characteristics}

While much attention has been devoted to the improvement of IR NDE images that TSR provides, there is a wealth of physical information that can be extracted from each pixel in a TSR data set. Unlike other IR NDE techniques that are based on evaluation of an image, either visually or computationally, it is possible to extract a significant amount of information from a single pixel, without reference to adjacent pixels or a human interpreter. The characteristics involved are invariant with respect to material or the specifics of the apparatus, and can be interpreted without a calibration standard or a priori knowledge of the target sample. To illustrate, it is useful to consider a large, adiabatically isolated slab of thickness $L$ that uniformly and instantaneously heated on its front surface. Plotting the front surface logarithmic temperature-time response and its first and $2^{\text {nd }}$ derivatives Fig. 1, we observe:

a. Although the log plot depends on the absorbed input energy and thermal properties of the sample, its shape, and hence its derivatives, remain invariant, and bounded within specified ranges.

b. As the thickness or the thermal diffusivity of the sample changes, the derivatives remain unchanged except for a shift left (thinner / higher diffusivity) or right (thicker / lower diffusivity).

c. The peak of the $2^{\text {nd }}$ derivative provides a precise indication of the time at which incident heat from the surface interacts with the back wall interface. 

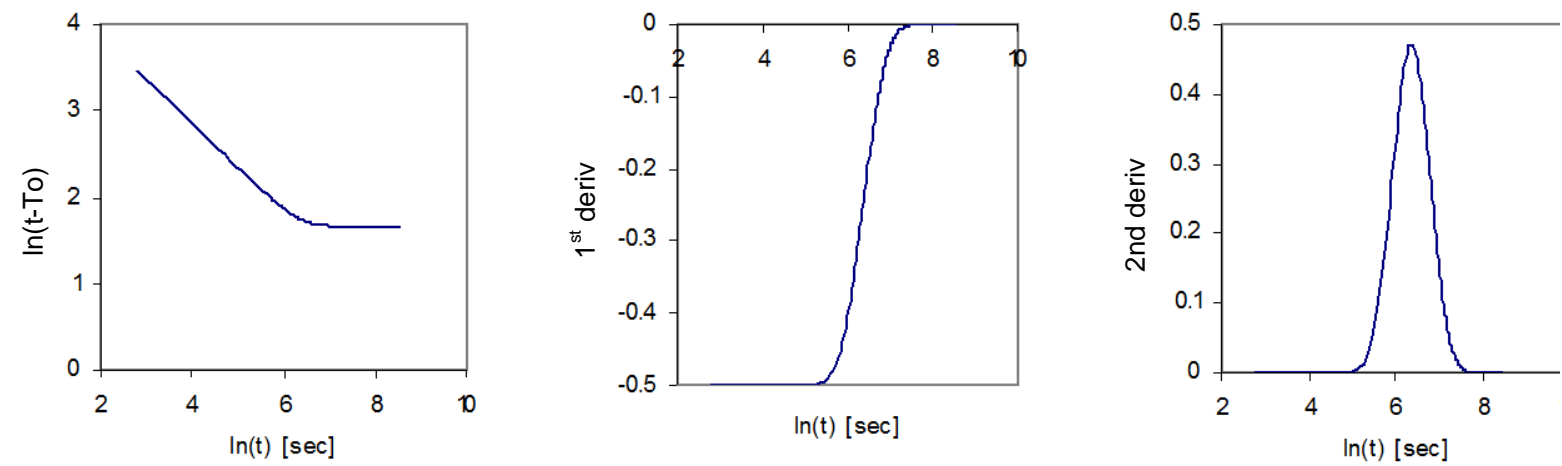

Figure 1(left to right) Logarithmic temperature. $1^{\text {st }}$ and $2^{\text {nd }}$ derivative plotted against In(time) for an adiabatic slab.

The $2^{\text {nd }}$ derivative for the adiabatic slab can be approximated to a high degree of accuracy with a Gaussian function. In the event that heat passes through the back wall, e.g. a multilayer system, the $2^{\text {nd }}$ derivative changes. For an infinitely thick substrate layer has lower thermal conductivity than the first layer, the peak derivative amplitude decreases, and it is followed by a negative-going component. In the case where the opposite conductivity conditions exist, the polarity of the $2^{\text {nd }}$ derivative changes correspondingly. In either case, the multilayer $2^{\text {nd }}$ derivative is bipolar, while the adiabatic slab derivative is uniquely positive, and in both cases, the derivative asymptotically approaches zero. However, in the case where the $2^{\text {nd }}$ layer has an adiabatically isolated back wall, the $2^{\text {nd }}$ derivative will make a sudden turn in the positive direction, regardless of the conductivity arrangement. It is important to recognize that we have made several inferences about the configuration of the sample from cursory examination of the $2^{\text {nd }}$ logarithmic time derivative of a single pixel:

a. Adiabatic slab (positive Gaussian)

b. Higher conductivity layer on thick substrate (positive peak followed by negative; 0 asymptote)

c. Lower conductivity layer on thick substrate (negative peak followed by negative; 0 asymptote)

d. Higher conductivity layer on substrate (positive peak followed by negative; positive asymptote)

e. Lower conductivity layer on thick substrate (negative peak followed by negative; positive asymptote)

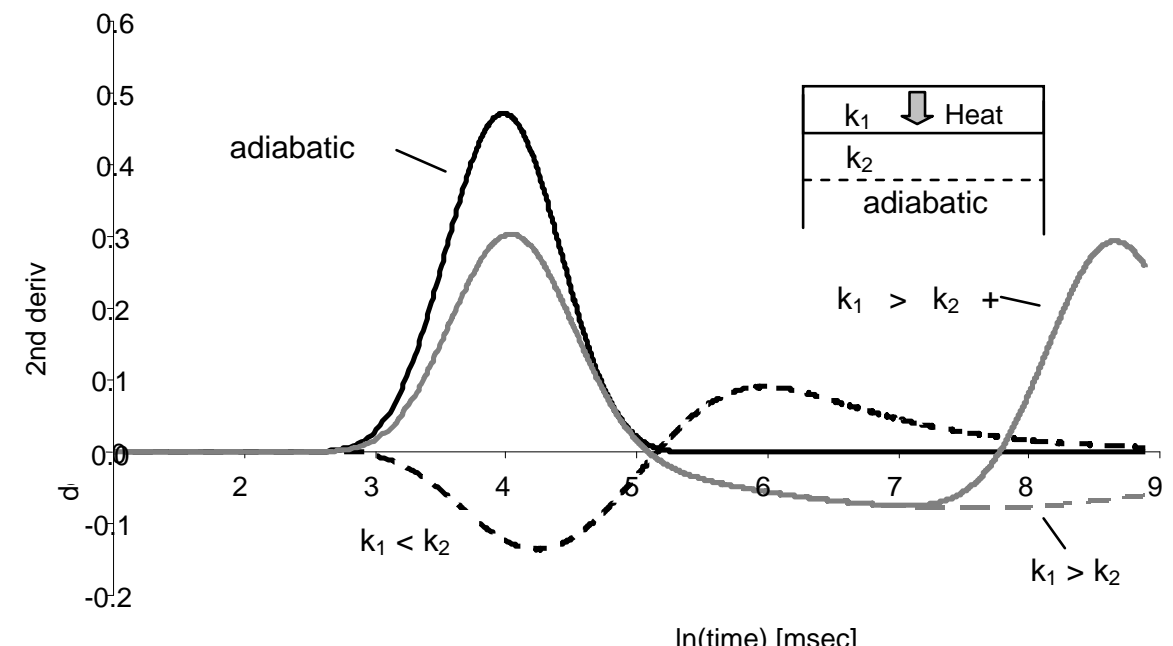

Figure 2: Multilayer behaviour of the $2^{\text {nd }}$ TSR derivative

We can make additional quantitative inferences by noting the time at which the $2^{\text {nd }}$ derivative maxima and zero crossings occur. It is also worth noting that we are discussing characterization of flaw-free samples, which would yield no signal whatsoever if contrast methods were employed. We will discuss the implications of the presence of a flaw on derivative behaviour, and additional quantitative information that can be derived from the derivatives in the full paper. 